\title{
ENTPD1 Gene
}

National Cancer Institute

\section{Source}

National Cancer Institute. ENTPD1 Gene. NCI Thesaurus. Code C113579.

This gene is involved in platelet activation. 